\title{
BMJ Open General populations' preferences for colorectal cancer screening: rationale and protocol for the discrete choice experiment in the SIGMO study
}

\author{
Melanie Brinkmann (D , ${ }^{1}$ Leonie Diedrich, ${ }^{1}$ Christian Krauth, ${ }^{1}$ Bernt-Peter Robra, ${ }^{2}$ \\ Jona Theodor Stahmeyer, ${ }^{3}$ Maren Dreier $^{1}$
}

To cite: Brinkmann M, Diedrich L, Krauth C, et al. General populations' preferences for colorectal cancer screening: rationale and protocol for the discrete choice experiment in the SIGMO study. BMJ Open 2021;11:e042399. doi:10.1136/ bmjopen-2020-042399

- Prepublication history for this paper is available online. To view these files, please visit the journal online (http://dx.doi. org/10.1136/bmjopen-2020042399).

Received 03 July 2020 Revised 17 December 2020 Accepted 30 December 2020

Check for updates

(C) Author(s) (or their employer(s)) 2021. Re-use permitted under CC BY-NC. No commercial re-use. See rights and permissions. Published by BMJ.

${ }^{1}$ Institute for Epidemiology, Social Medicine and Health Systems Research, Hannover Medical School, Hannover, Germany

${ }^{2}$ Institute of Social Medicine and Health Systems Research, Otto-von-Guericke University Magdeburg, Magdeburg,

Germany

${ }^{3}$ Health Services Research Unit, AOK Niedersachsen, Hannover, Germany

Correspondence to Melanie Brinkmann; Brinkmann.Melanie2@mhhannover.de

\begin{abstract}
Introduction In Germany, the organised colorectal cancer (CRC) screening programme includes the immunologic faecal occult blood test and colonoscopy. The sigmoidoscopy is recommended for individuals rejecting colonoscopy but is not included into the screening programme. To examine whether the evidence based sigmoidoscopy should be additionally offered, the first objective of this study is to evaluate the demand for sigmoidoscopy by analysing the German general populations' preferences for CRC screening.
\end{abstract}

Methods and analysis Preference data will be collected using a discrete choice experiment (DCE). Identification and selection of the attributes and their levels will be supported by evidence resulting from a systematic literature search and focus groups. An efficient, fractional factorial choice design will be generated. In a cross-sectional study, the DCE will be administered as a written questionnaire to a random sample of 4000 members of the statutory health insurance company in Lower Saxony (AOK Lower Saxony). Insured persons 50-60 years of age without CRC or a chronic inflammatory bowel disease will be eligible. The collected choice data will be analysed by conducting a conditional logit regression model and latent class models. Ethics and dissemination Ethical approval for this study was obtained from the Ethics Committee of Hannover Medical School (reference number 8671_B0_K_2019). The study results will be disseminated via conference presentations, publications in peer-reviewed journals and, to participants, the membership magazine of the AOK Lower Saxony.

Trial registration number DRKS00019010.

\section{INTRODUCTION}

In 2012, colorectal cancer (CRC) was the second most common cancer in Europe with an incidence of almost 447000 for both sexes and the second leading cause for cancer related deaths, resulting in approximately 215000 deaths. ${ }^{1}$ In Germany, the agestandardised CRC incidence and mortality rates for both women and men are slightly declining. ${ }^{2}$ However, CRC was still the second and third most frequent cancer for women (25990 new cases) and men (32 300) as well
Strengths and limitations of this study

To the best of our knowledge, this study is the first discrete choice experiment that evaluates the preferences of the German general population for colorectal cancer screening.

- This study will provide insight into the attributes and attribute levels that influence decisions for or against colorectal cancer screening measures, and the trade-offs made between the attributes.

- We will use a mix of systematic literature search, qualitative research and expert consultation to identify relevant attributes and attribute levels. It cannot be ruled out, however, that we will miss characteristics of importance for our target group.

- AOK Lower Saxony is the largest health insurance company in the federal state of Lower Saxony with 2.85 million insurees and $38 \%$ market share, however, the insured population differs with respect to education and occupation from the German general population which has to be taken into account when generalising the results of this study.

as the third leading cause for cancer-related deaths for both sexes in $2016 .^{2}$

The vast majority of CRCs arises out of precancerous stages (adenoma-carcinoma sequence) over a period of several years. ${ }^{3}$ Due to the possibility to detect and remove adenomas, endoscopic methods such as the colonoscopy and the sigmoidoscopy can disrupt the potential adenoma-carcinoma sequence and, subsequently, not only detect CRC but also prevent its development. ${ }^{34}$ Evidence of the effect of the sigmoidoscopy on CRC incidence and mortality compared with no screening was based on a meta-analysis of four randomised controlled trials showing a relative reduction in incidence of $24 \%$ (RR $0.76 ; 95 \%$ CI 0.70 to 0.83 ) and a relative reduction in CRC mortality of $26 \%$ (RR $0.74 ; 95 \%$ CI 0.69 to 0.80$).{ }^{5}$ For colonoscopy, a relative reduction in CRC incidence of $69 \%$ (RR $0.31 ; 95 \%$ CI 0.12 to 0.77 ) and mortality of $68 \%$ 
Table 1 Summary of the organised screening programme for colorectal cancer for statutory insured persons in Germany

听

Once-only consultation by physician about screening programme for CRC (from the age of 50 years)

网

Gender-specific information about CRC screening and a written invitation for participation (at the ages of $50,55,60$ and 65 )

\begin{tabular}{|c|c|c|c|c|c|c|c|c|}
\hline \multirow[b]{2}{*}{$\begin{array}{c}\text { 可 } \\
\text { iFOBT }\end{array}$} & \multicolumn{2}{|c|}{ 50-54 years } & \multicolumn{2}{|c|}{55 years or older } & \multicolumn{2}{|c|}{$50-54$ years } & \multicolumn{2}{|c|}{55 years or older } \\
\hline & 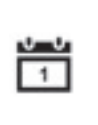 & Annually & بـ & Biennially & 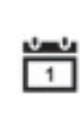 & Annually & 4 & Biennially \\
\hline & \multicolumn{2}{|r|}{-} & \multicolumn{2}{|c|}{ OR } & \multicolumn{2}{|c|}{ OR } & \multicolumn{2}{|c|}{ OR } \\
\hline Colonoscopy & प & Not available & 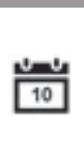 & $\begin{array}{l}\text { Twice at an } \\
\text { interval of } \\
10 \text { years }\end{array}$ & 40 & $\begin{array}{l}\text { Twice at an } \\
\text { interval of } \\
10 \text { years }\end{array}$ & & $\begin{array}{l}\text { Twice at an } \\
\text { interval of } \\
10 \text { years }\end{array}$ \\
\hline
\end{tabular}

${ }^{*} \mathrm{~A}$ maximum of two colonoscopies for $\mathrm{CRC}$ screening purposes will be offered. A colonoscopy at 65 years or older isconsidered as second colonoscopy.

CRC, colorectal cancer; iFOBT, immunologic faecal occult blood test.

(RR 0.32; 95\% CI 0.23 to 0.43 ) was estimated. ${ }^{6}$ The effects of the screening colonoscopy, however, are based on observational studies only because data from randomised controlled trials are not available up to now. ${ }^{7}$

The risk of serious adverse events per 10000 screening colonoscopies is 4 (2.4-5.4) for perforations and 8 (4.913.6) for bleedings. ${ }^{3}$ A colonoscopy is associated with an increased effort resulting from bowel cleansing with laxatives starting the day before the examination, sedation usually administered requiring a companionship for the way home, and an inability to work on the day of the examination. ${ }^{8-10}$ For sigmoidoscopy, perforations occur in $1(0.4-1.4)$ per 10000 screening procedures and the risk of bleedings is $2(0.7-4.4) .^{3}$ Bowel preparation usually includes an enema immediately before the examination and sedation is usually not necessary. ${ }^{10-12}$

In Germany, an organised, quality-assured screening programme for CRC was introduced in 2019. ${ }^{13}$ Statutory insured persons aged 50 years or older can decide between the immunologic faecal occult blood test (iFOBT) and the colonoscopy for CRC screening depending on their sex and age. ${ }^{13}$ The screening programme also includes written invitation and evidence-based information material to enhance informed decision making whether to participate or not. ${ }^{13} 14$ A comprehensive summary of the organised screening programme for CRC is given in table 1 .

With about $22 \%$ and $20 \%$ of the eligible women and men that chose the colonoscopy for CRC screening within the first 10 years after its implementation in 2002, participation rates are rather low. ${ }^{15}$ In addition, only every seventh women $(15.1 \%)$ and every ninth men $(11.4 \%)$ eligible for participation used the guaiac-based FOBT (gFOBT) in 2014 (iFOBT was introduced in 2017). ${ }^{16}$

The sigmoidoscopy is recommended for individuals rejecting the screening colonoscopy but is not included into the screening programme. ${ }^{4}$ In contrast, some European countries offer the sigmoidoscopy as a CRC screening method. The English Bowel Cancer Screening Programme using faecal occult blood testing was extended by a once-only sigmoidoscopy (bowel scope screening) at the age of 55 in 2013. ${ }^{17}$ In the Italian region Piedmont, the sigmoidoscopy is available once for people between 58 and 60 years of age. ${ }^{18}$

Taking into account that the sigmoidoscopy is an evidencebased procedure with lower risks and a reduced effort compared with colonoscopy and that participation is low, particularly in the endoscopic colonoscopy, the question must be raised whether additionally offering the sigmoidoscopy for CRC screening could improve healthcare. Possible target groups for the sigmoidoscopy could be individuals refusing the colonoscopy or people for whom the colonoscopy is accompanied with an increased risk due to the effort associated with the procedure. To enhance the acceptance of healthcare interventions and treatments, decisions regarding the organisation of healthcare and the allocation of healthcare resources have to consider the general populations' preferences for the alternatives that are available. ${ }^{1920}$ Being aware of populations' preferences helps to understand their needs and expectations. ${ }^{21}$ One method to elicit preferences as an expression of utility associated with the components of a specific intervention or healthcare strategy is a discrete choice experiment (DCE) located in economic theory. ${ }^{21}$

\section{OBJECTIVES}

This protocol describes the first part (DCE) of the 'Sigmoidoscopy as an evidence based colorectal cancer screening test-a possible option?' (SIGMO: Die Sigmoidoskopie als evidenzbasiertes Verfahren zur Darmkrebsfrüherkennung-eine mögliche Option?) study in detail. The objectives are: 
- To analyse the relative importance of the attribute levels and the utility associated with the attributes of methods for CRC screening for the German general population and to examine if the preferences are depending on sociodemographic characteristics.

- To examine the (hypothetical) CRC screening method that is preferred by the German general population.

Second part of the SIGMO study is a decision-analytic modelling approach (decision tree and Markov model) analysing the cost-effectiveness of standard care extended by offering the sigmoidoscopy as a CRC screening method compared with the screening programme currently offered in Germany. The protocol of the second part will be published elsewhere.

\section{METHODS AND ANALYSIS}

\section{Method and theory of discrete choice experiments}

In contrast to revealed preference data that give information about choices actually made and observed, DCEs are used in health economics to collect and evaluate stated preference data for hypothetical health-related products or programmes within an experimental approach. ${ }^{192} 23$ Individuals receive several choice sets (or choice tasks) consisting of two or more hypothetical alternatives that are defined by a given number of characteristics (attributes) and differ in their attribute levels from each other. ${ }^{19}{ }^{23}$ For preference elicitation, individuals should decide between the hypothetical alternatives of each choice set and choose the preferred one. ${ }^{1924}$

The analysis of the choices made in DCEs is mainly based on the assumptions of Lancaster's economic theory of value ${ }^{25}$ and on McFadden's extended work on random utility theory (RUT). ${ }^{26}{ }^{27}$ According to Lancaster, individuals derive utility not from a good (alternative) but from the attributes it is composed of. ${ }^{25}$ Assuming that individuals choose the alternative associated with the highest utility, changes in these attributes can be accompanied by a shift in decision making to a more preferred alternative. ${ }^{192025}$ Therefore, DCEs provide insight into the relative utility that individuals associate with the attribute levels (part-worth utilities), the overall utility of an alternative resulting in the sum of the part-worth utilities, and the trade-offs that individuals make between attributes. ${ }^{192}$ RUT defines utility as an additive function decomposable in a (1) systematic, explainable and observable component meaning the attributes of the chosen alternatives and a (2) random, unexplainable and unobservable component including, for example, measurement errors or attributes that were not considered in the definition of the hypothetical alternatives but also affect individual choices. ${ }^{19} 2028$ Under the assumption that individuals' utility for a good cannot entirely be observed or measured, overall utility is latent. ${ }^{19} 27$

Following the recommendations of the International Society for Pharmacoeconomics and Outcomes Research (ISPOR) for applying DCEs in healthcare, ${ }^{29}$ the preference elicitation process in the SIGMO study can be subdivided in (1) the identification and selection of relevant attributes and attribute levels supported by the results of (A) a systematic literature search and (B) focus groups, (2) creating the choice tasks and developing the experimental design, (3) collecting the data including sampling the study population and (4) analysing the choice data.

\section{Identification and selection of attributes \\ Systematic literature search}

To acquire an evidenced based overview of all attributes with possible relevance for the characterisation of our choice context, ${ }^{19}$ we will conduct a sensitive systematic literature search ${ }^{30}$ in the bibliographic databases PubMed, Embase, Biomedical Reference Collection: Corporate Edition, Web of Science, PsycINFO and LIVIVO. Additional studies will be identified by checking the reference lists of the included studies. The search strategy will be a combination of database specific controlled vocabulary search terms and various text terms including their synonyms. Eligible studies are conjoint analyses or DCEs considering at least one of the following CRC screening tests: stool tests (gFOBT, iFOBT), colonoscopy and sigmoidoscopy. English language reports published since 1 January 2000 or later will be included. This date restriction was applied due to actuality reasons and because the application of DCEs to health-related questions started in the 1990 s. ${ }^{19}$

\section{Focus groups}

In accordance with the ISPOR recommendations to use a mix of quantitative and qualitative research methods for the identification and selection of relevant attributes, ${ }^{24} 29$ we will conduct four focus groups with a sample of the German general population in addition to the systematic literature search. This allows us to analyse the level of importance of the identified attributes for our target population, and to identify further attributes with potential relevance for choices made in the context of CRC screening from their point of view. To achieve this and to ensure that the same questions will be asked in all focus groups, we will develop and pilot test a questioning route before running the first focus group. ${ }^{31}$ Individuals aged 50-60 years old without ever being diagnosed with CRC and without a diagnosed bowel disease associated with an increased risk for CRC such as ulcerative colitis or Crohn's disease will be eligible for participation. The recruitment of participants will take place via outpatient clinics of the Hannover Medical School and medical practices. Participants will get $€ 50$ as a compensation of the effort associated with their participation. To gain a broad range of opinions and possible attributes, the composition of the four focus groups will be made on the basis of the criteria sex and previous experience with colonoscopy. Each focus group will comprise five to eight persons and will take $90-120 \mathrm{~min}$. The focus groups will be audiorecorded, transcribed and after importing the written qualitative data in MAXQDA (VERBI Software, Berlin, Germany), analysed using content analysis. 
Table 2 Example of a generic choice set

\begin{tabular}{lcc}
\hline Attributes & Alternative A & Alternative B \\
\hline Preparation & Enema & None \\
Pain & No & Mild \\
Colorectal cancer deaths & 5 out of 1000 & 0 out of 1000 \\
New colorectal cancer & 2 out of 1000 & 10 out of 1000 \\
cases & & No \\
Need for transportation & Yes & 10 years \\
Screening interval & 2 years & $\square$ \\
\hline I choose & $\square$ & $\square$ \\
\hline
\end{tabular}

\section{Creating the choice tasks and developing the experimental} design

Based on the results generated from the systematic literature search and the focus groups, the final list of attributes will be consented in our multidisciplinary team and the number and range of levels that are assigned to each of them will be generated. ${ }^{29}$ Level selection will be based on current high-quality evidence and the information material provided in the context of the organised screening programme for CRC.

Attributes can be quantitative (eg, screening interval) or qualitative (eg, preparation), even though there is a risk that qualitative level labels may be interpreted differently by the respondents. ${ }^{19} 2324$ The alternatives can be named in an alternative-specific (eg, colonoscopy, sigmoidoscopy) or a generic (eg, alternative A, alternative B) way. ${ }^{19}{ }^{24}$ While alternative specific labels reduce cognitive burden by creating familiarity with the presented products or programmes and suggesting realistic decision contexts, it is possible that the respondents' choices will be based on or influenced by the associations evoked by the labels instead of resulting from weighing up the attributes and attribute levels of the contrasted alternatives. ${ }^{1924}$ An example choice set is given in table 2 .

With $\mathrm{A}$ attributes and $\mathrm{L}$ levels, the maximum number of possible combinations or alternatives, also called as the full factorial design, is equivalent to $\mathrm{L}^{\mathrm{A}} \cdot{ }^{19}$ As an example, the inclusion of six attributes $(A=6)$ with three levels each $(\mathrm{L}=3)$ results in $3^{6}=729$ possible level combinations (full factorial design) from which $\mathrm{L}^{\mathrm{A}} *\left(\mathrm{~L}^{\mathrm{A}}-1\right) / 2=(729 *$ 728) $/ 2=265356$ possible choice sets consisting of two alternatives each could be generated. ${ }^{19}{ }^{24}$ Besides the number of attributes defining the alternatives and the number of alternatives within each choice set, the complexity of choices that have to be made from respondents also depends, for example, on the number of choice sets each participant has to evaluate. ${ }^{19}$ To increase practicability and to reduce the number of choice tasks presented to each respondent as well as cognitive complexity, (1) using a fractional factorial design (subset of all possible combinations) and/or (2) blocking the experimental design into various versions with the same number of choice tasks each from which each participant will only receive one could be considered. ${ }^{19232429}$
According to Huber and Zwerina, ${ }^{32}$ an efficient choice design should meet the following criteria: level balance, orthogonality, minimal overlap and utility balance. Level balance and orthogonality in an experimental design mean that each level of an attribute occurs equally often (=level balance) and each pair of attribute levels of all twoway combinations of the included attributes appears with equal frequency (=orthogonality). ${ }^{232432}$ Minimal overlap refers to situations where all alternatives within a choice task are characterised by the same level of one attribute resulting in the fact that no information can be gained about the utility associated with the respective attribute. ${ }^{32}$ Therefore, the occurrence of such choice sets in an experimental design should be reduced to a minimum. The alternatives within a choice task should be of comparable appeal for the participants (=utility balance). ${ }^{23}{ }^{24}$ Choice sets with one alternative being clearly better across all attributes (=dominating alternative) cannot reveal any information about trade-offs made between the hypothetical alternatives. ${ }^{19}$ However, such a choice task is useful to test whether the participants understand the DCE, which is important to estimate the internal validity of the data generated by the DCE. ${ }^{24} 33$

To ensure that the choices made by the participants will not be influenced by the labels of the alternatives but are based on weighing up the included attributes, we will develop a generic, unlabelled DCE. This should also prevent irritation among participants wondering why they have not been offered the sigmoidoscopy for CRC screening. To keep the cognitive burden for respondents low, we intend to include four to eight attributes ${ }^{34}$ and limit the number of choice tasks to a maximum of 16 to 20 per participant. ${ }^{192429}$ In addition, we will explain the choice tasks and the included attributes and attribute levels in detail in the introductory section to the DCE of the data collection instrument to ensure equal understanding among participants and to prevent that other, unobservable aspects would be considered in decision making. ${ }^{29}$

Taking into account the criteria explained above, an efficient, fractional factorial choice design will be constructed using the dcreate command in the statistic software Stata (StataCorp V.15). To check the responses on rationality and consistency, a dominant choice set will be included additionally to the experimental design but will not be considered in the analysis of the choice data. ${ }^{19}$

Before starting to collect the choice data, the understanding of the choice tasks, of the descriptions of the attributes and attribute levels as well as the amount of complexity associated with the evaluation of the choice sets will be pilot tested and corrected if necessary ${ }^{23} 29$ by cognitive interviews with eight to ten individuals. ${ }^{35}$

\section{Collecting the choice data including sampling the study population}

We will survey a random sample of individuals who received a written invitation to participate in the national CRC screening programme from their health insurance 
company (AOK Lower Saxony). Insurees eligible for our study are 50-60 years old and capable to complete a questionnaire in German language. The age restriction allows us to compare the preferences between people who had never been (50-54year olds) and who had already been (55-60year olds) eligible for colonoscopy as a CRC screening test. Subjects older than 60 years will be excluded because we want to evaluate if sigmoidoscopy can be an alternative for individuals who reject screening colonoscopy in the first place. Insured persons with CRC or a chronic inflammatory bowel disease will be excluded from our study. Diagnoses are based on claims data from the health insurance company. Claims data are routinely collected by statutory health insurance companies for billing purposes and form the basis to draw the random sample in our study. Data contain, for example, sociodemographic information and diagnoses from inpatient and outpatient services that are coded by using the German modification of the International Statistical Classification of Diseases and Related Health Problems (ICD-10-GM) ${ }^{36}$ Insured persons that meet one of the exclusion criteria will be identified in advance by the health insurance company using defined ICD-10-GM codes and will not be considered in the sampling procedure.

Participants receive a written, self-administered postal questionnaire by their health insurance company consisting of (1) the DCE, (2) questions on the respondents' intention to participate in CRC screening tests and (3) questions on sociodemographic characteristics and health-related information including previous experience with colonoscopy.

Our sample size calculations are based on the equation $\frac{n t a}{c} \geq 1000$ from Johnson and Orme ${ }^{37}$ according to which the minimal number of individuals to include (n) depends on the number of choice tasks (t), on the number of alternatives within each choice set (a) and on the highest number of attribute levels across all attributes (c). With subject to the final list of attributes and attribute levels, the minimum sample size with $\mathrm{t}=6, \mathrm{a}=2$ and $\mathrm{c}=3$ requires $\mathrm{n}=250$ respondents. To allow for subgroup analyses by sex and previous experience with colonoscopy, the minimum sample size increases up to $n=1000$. With an estimated response rate of $25 \%-35 \%$, a total of 4000 insured persons will be invited for participation. To increase the response, the questionnaires will be sent once again after 3 weeks.

\section{Analysing the choice data}

After cleaning and preparing the collected data that also includes the conversion of the data set to a format required for the statistical analysis of choice data, ${ }^{38}$ descriptive analyses will be conducted. To estimate the preferences associated with the attributes and attribute levels, a conditional logit regression model will be used to analyse the choice data obtained from the DCE. ${ }^{39}$ The resulting coefficients (preference weights) reflect the contribution of the included levels to the utility associated with an alternative. ${ }^{39}$ They provide information on
(1) the attributes and levels that are considered (or not) in choosing one of the alternatives (significance level), (2) more or less preferred levels of an attribute (sign and height of coefficients) and (3) the relative importance of an attribute over the range of the respective level coefficients for decision making. ${ }^{19} 3839$ The coefficients of a conditional logit regression model, however, represent a mean effect over the sample only, and do not account for possible preference heterogeneity resulting from, for example, differences in sociodemographic characteristics. ${ }^{19}{ }^{39}$ To account for preference variation among respondents and to avoid biased interpretation of preference weights from the conditional logit regression model, a latent-class model will be performed resulting in a predefined number of classes of individuals with similar preferences. Respondents who did not complete at least one choice set will be excluded from the analyses. The statistic software Stata (StataCorp V.15) will be used to analyse the choice data generated by the DCE. Additionally, to compare the planned uptake stated in the questionnaire with the actual uptake documented in the claims data, we will conduct a record linkage between the claims data and the self-reported data. ${ }^{40}$

The preferences resulting from this DCE will be incorporated as one data input source among others in the decision-analytic modelling approach. For this purpose, the attribute levels are combined in such a way that the three screening alternatives stool test, sigmoidoscopy and colonoscopy are represented as realistically as possible. Taking into account the preference weights of the respective attribute levels, indirect utility associated with each of these screening methods and the probability of uptake can be estimated. ${ }^{19}$ The probabilities for take-up will then be incorporated into the modelling approach.

\section{DISCUSSION}

To the best of our knowledge, this is the first DCE analysing the preferences of the German general population for CRC screening. As a result, information is expected about the CRC screening test preferred by the respondents and whether there is a subgroup favouring sigmoidoscopy. If the decision-analytic modelling approach also concludes that the additional offer of the sigmoidoscopy can improve medical care for, for example, a subpopulation, the next step could be to initiate a pilot project in a network of general practitioners and gastroenterologist with offering the sigmoidoscopy as an alternative to stool testing to individuals rejecting the colonoscopy.

A strength of this study is that the identification of relevant attributes will not be based on a systematic literature search only, but also takes into account the views of the target population by conducting focus groups. The selection of the final attributes, however, will be based on weighing up the results of the systematic literature search and the focus groups, the research question and recommendations regarding the optimal number of attributes to be included for characterising the choice tasks. It is, 
therefore, possible, that not all attributes with importance for the respondents can be considered. Cognitive pilot tests may indicate whether relevant attributes are missing or attributes with no influence on decision making are included, so that changes can be made if necessary.

Another methodological challenge may be the representativeness of the population insured with the AOK Lower Saxony. A study ${ }^{41}$ showed that the insurees differ with respect to education and occupation from the German general population. This needs to be investigated. Possible deviations will be accounted for by weighting, if necessary.

Finally, participation bias can occur. To optimise the response rate, the questionnaire and cover letter will be designed appealing, clear and easy to understand, and a telephone contact will be offered. In addition, the understanding of the DCE will be tested by cognitive interviews. It is, however, possible, that the participants will deviate from the selected random sample. To evaluate the response rate according to age, gender and occupational status, the AOK Lower Saxony will conduct a non-responder analysis. Based on the results, the study population will be adjusted for different characteristics, if necessary.

\section{Patient and public involvement}

No patients were involved in the development of the research question and the design of this study. To consider characteristics of importance for the target population when selecting the final list of attributes to describe the choice tasks, individuals eligible for CRC screening will be involved by conducting focus groups. For data collection, insurees of the AOK Lower Saxony will be recruited. The results will be disseminated to the participants in the membership magazine of the health insurance company.

\section{ETHICS AND DISSEMINATION}

The research protocol including a data protection concept, the informed consent forms, participant information materials and the questioning route for the focus groups were reviewed and approved by the Ethics Committee of Hannover Medical School (reference number 8671_BO_K_2019). The inclusion and exclusion criteria will be applied by the AOK Lower Saxony to meet data security legal requirements. Insurees will be informed that participation is completely voluntary and that they have the right to refuse or withdraw at any time without any disadvantages. Participation will be based on informed consent. To ensure confidentiality of the collected data, the health insurance company will know the insured persons who have not replied, but will not receive any information from the completed questionnaires which will remain and be stored at the Hannover Medical School. This allows the AOK Lower Saxony to follow up non-participants with a replacement questionnaire and to conduct a non-responder analysis to check for sample representativeness and participation bias.
On the other hand, the research team at the Hannover Medical School will not get any information that enables personal identification of the participants. In accordance with Article 75, Book 10 of the Code of Social Law, the transfer of the claims data for our research purpose was approved by the Ministry for Social Affairs of Lower Saxony on 4 December 2019.

Focus groups participants will also be informed about the voluntariness of their participation and their right to refuse or withdraw without any disadvantages. The audio recorded data will be transcribed and analysed without personal information and deleted after transcription is completed.

The results of this study will be disseminated via publications in peer-reviewed journals, conference presentations and, to participants, in the membership magazine of the AOK Lower Saxony. Additionally, the study results will be communicated to the funder by annual progress reports and a final report within 6 months after completion of the study.

Contributors Conceptualisation: CK, JTS and MD. Funding acquisition: CK, JTS and MD. Methodology: MB, CK, JTS and MD. Project administration: CK, JTS and MD. Supervision: CK, B-PR, JTS and MD. Visualisation: MB. Writing-original draft: MB. Writing-review and editing: MB, LD, CK, B-PR, JTS and MD. All authors have provided input to, reviewed, edited and approved the final version.

Funding This study is funded by the German Federal Joint Committee's Innovation Fund (grant number 01VSF18007) from 1 March 2019 to 28 February 2022.

Disclaimer The funder had no role in study design, data collection and analysis, interpretation of the data, decision to submit the protocol or results for publication, or writing the manuscript.

Competing interests All authors have completed the ICMJE uniform disclosure form at www.icmje.org/coi_disclosure.pdf and declare: MB, LD, CK, JTS and MD had financial support from the German Federal Joint Committee's Innovation Fund (grant number 01VSF18007). B-PR had no financial support from any organisation for the submitted work; all authors had no financial relationships with any organisations that might have an interest in the submitted work in the previous three years; no other relationships or activities that could appear to have influenced the submitted work.

Patient and public involvement Patients and/or the public were involved in the design, or conduct, or reporting, or dissemination plans of this research. Refer to the Discussion section for further details.

Patient consent for publication Not required.

Provenance and peer review Not commissioned; externally peer reviewed.

Open access This is an open access article distributed in accordance with the Creative Commons Attribution Non Commercial (CC BY-NC 4.0) license, which permits others to distribute, remix, adapt, build upon this work non-commercially, and license their derivative works on different terms, provided the original work is properly cited, appropriate credit is given, any changes made indicated, and the use is non-commercial. See: http://creativecommons.org/licenses/by-nc/4.0/.

ORCID iD

Melanie Brinkmann http://orcid.org/0000-0003-2636-2328

\section{REFERENCES}

1 Ferlay J, Steliarova-Foucher E, Lortet-Tieulent J, et al. Cancer incidence and mortality patterns in Europe: estimates for 40 countries in 2012. Eur J Cancer 2013;49:1374-403.

2 Robert Koch Institute, Association of Population-based Cancer Registries in Germany. Cancer in Germany in 2015/2016, 2019. Available: www.krebsdaten.de/english [Accessed 16 Jun 2020].

3 Lin JS, Piper MA, Perdue LA. Screening for colorectal cancer: an updated systematic review for the U.S. preventive services Task 
force: evidence syntheses No. 135. AHRQ publication No. 14-05203EF-1, 2015. Available: https://www.uspreventiveservicestaskforce. org/Home/GetFile/1/685/coloncandraftes135/pdf [Accessed 12 Dec 2019].

4 German Cancer Society, German Cancer Aid, AWMF (German Guideline Program in Oncology). S3-guideline colorectal cancer, long version 2.1, 2019, AWMF registrationnumber: 021-007OL, 2019. Available: https://www.leitlinienprogramm-onkologie.de/leitlinien/ kolorektales-karzinom/ [Accessed 16 Jun 2020].

5 Jodal HC, Helsingen LM, Anderson JC, et al. Colorectal cancer screening with faecal testing, sigmoidoscopy or colonoscopy: a systematic review and network meta-analysis. BMJ Open 2019;9:e032773 https://bmjopen.bmj.com/content/bmjopen/9/10/ e032773.full.pdf

6 Brenner H, Stock C, Hoffmeister M. Effect of screening sigmoidoscopy and screening colonoscopy on colorectal cancer incidence and mortality: systematic review and meta-analysis of randomised controlled trials and observational studies. BMJ 2014;348:g2467 https://www.bmj.com/content/bmj/348/bmj.g2467. full.pdf

7 Lauby-Secretan B, Vilahur N, Bianchini F, et al. The IARC perspective on colorectal cancer screening. N Engl J Med 2018;378:1734-40.

8 Senore C, Ederle A, Fantin A, et al. Acceptability and side-effects of colonoscopy and sigmoidoscopy in a screening setting. $J$ Med Screen 2011;18:128-34 https://journals.sagepub.com/doi/

9 Ball AJ, Rees CJ, Corfe BM, et al. Sedation practice and comfort during colonoscopy: lessons learnt from a national screening programme. Eur J Gastroenterol Hepatol 2015;27:741-6 https:// insights.ovid.com/article/00042737-201506000-00020

10 Helsingen LM, Vandvik PO, Jodal HC, et al. Colorectal cancer screening with faecal immunochemical testing, sigmoidoscopy or colonoscopy: a clinical practice guideline. BMJ 2019;367:15515 https://www.bmj.com/content/367/bmj.I5515

11 Maliampurakal AJ, McMillan DC, Anderson JH, et al. Factors associated with the efficacy of polyp detection during routine flexible sigmoidoscopy. Frontline Gastroenterol 2018;9:135-42.

12 Cespedes I, Seidler U, Walter U, et al. Physicians' view on sigmoidoscopy as an additionally offered method for colorectal cancer screening. Z Gastroenterol 2019;57:1059-66 https://www. thieme-connect.de/products/ejournals/abstract/

13 Federal Joint Committee. Directive on organized cancer screening programs, 2020. Available: https://www.g-ba.de/richtlinien/104/ [Accessed 15 Jun 2020].

14 Institute for Quality and Efficiency in Health Care. Invitation and decision aid for bowel cancer screening. final report, 2016. Available: https://www.iqwig.de/en/projects-results/projects/health-information/ p15-01-invitation-and-decision-aid-for-bowel-cancer-screening. 6620.html [Accessed 9 Dec 2020].

15 Brenner H, Altenhofen L, Stock C, et al. Prevention, early detection, and overdiagnosis of colorectal cancer within 10 years of screening colonoscopy in Germany. Clin Gastroenterol Hepatol 2015;13:717-23.

16 Zentralinstitut für die kassenärztliche Versorgung in der Bundesrepublik Deutschland. Projekt: Wissenschaftliche Begleitung von Früherkennungs-Koloskopien in Deutschland. Berichtszeitraum 2014. 12. Jahresbericht, version 2, Im Auftrag des GKVSpitzenverbands und Der Kassenärztlichen Bundesvereinigung, 2016. Available: https://www.zi.de/forschungsgebiete/studien/ darmkrebs-frueherkennung/ [Accessed 9 Dec 2020].

17 Atkin W, Wooldrage K, Parkin DM, et al. Long term effects of onceonly flexible sigmoidoscopy screening after 17 years of follow-up: the UK flexible sigmoidoscopy screening randomised controlled trial. Lancet 2017;389:1299-311.

18 Ponti A, Anttila A, Guglielmo R. Cancer screening in the European Union. Report on the implementation of the Council recommendation on cancer screening, 2017. Available: https://ec.europa.eu/ health/sites/health/files/major_chronic_diseases/docs/2017_ cancerscreening_2ndreportimplementation_en.pdf [Accessed 1 Oct 2019].

19 Ryan M, Gerard K, Amaya-Amaya M. Using discrete choice experiments to value health and health care. Springer: Dordrecht, 2008.

20 Mühlbacher A, Bethge S, Tockhorn A. Measuring preferences in healthcare: introduction to discrete-choice experiments]. Gesundh ökon Qual manag 2013;18:159-72.
21 Bridges JFP, Onukwugha E, Johnson FR. Patient preference methods - a patient centered evaluation paradigm. ISPOR Connections, 2007: 4-7. https://www.ispor.org/docs/default-source/ sig-documents/Patient-Preference-Methods.pdf

22 Hensher DA, Rose JM, Greene WH. Applied choice analysis: a primer. Cambridge: Cambridge University Press, 2005.

23 Lancsar E, Louviere J. Conducting discrete choice experiments to inform healthcare decision making: a user's guide. Pharmacoeconomics 2008;26:661-77 https://link.springer.com/ article/10.2165\%2F00019053-200826080-00004

24 Mühlbacher A, Johnson FR. Choice experiments to quantify preferences for health and healthcare: state of the practice. Appl Health Econ Health Policy 2016;14:253-66.

25 Lancaster KJ. A new approach to consumer theory. J Polit Econ 1966;74:132-57 https://www.jstor.org/stable/1828835

26 McFadden D. Conditional logit analysis of qualitative choice behavior. In: Zarembka P, ed. Frontiers in econometrics. New York: Academic Press, 1974: 105-42.

27 Louviere JJ, Flynn TN, Carson RT. Discrete choice experiments are not conjoint analysis. Journal of Choice Modelling 2010;3:57-72 https://www.sciencedirect.com/science/article/pii/ S1755534513700149?via\%3Dihub

28 de Bekker-Grob EW, Ryan M, Gerard K. Discrete choice experiments in health economics: a review of the literature. Health Econ 2012;21:145-72.

29 Bridges JFP, Hauber AB, Marshall D, et al. Conjoint analysis applications in health-a checklist: a report of the ISPOR good research practices for conjoint analysis task force. Value Health 2011;14:403-13.

30 Higgins JPT, Thomas J, Chadler J. Cochrane handbook for systematic reviews of interventions. 2nd edn, 2019. https://training. cochrane.org/handbook/current

31 Morgan DL, Krueger RA. The focus group kit: volumes 1-6. Thousand Oaks: SAGE Publications, 1998.

32 Huber J, Zwerina K. The importance of utility balance in efficient choice designs. J Market Res 1996;33:307-17 https://faculty. fuqua.duke.edu/ jch8/bio/Papers/Huber\%20Zwerina\%201996\% 20Marketing\%20Research.pdf

33 Johnson FR, Yang J-C, Reed SD. The internal validity of discrete choice experiment data: a testing tool for quantitative assessments. Value Health 2019;22:157-60.

34 Soekhai V, de Bekker-Grob EW, Ellis AR, et al. Discrete choice experiments in health economics: past, present and future. Pharmacoeconomics 2019;37:201-26.

35 Beatty PC, Willis GB. Research synthesis: the practice of cognitive interviewing. Public Opin Q 2007;71:287-311 https://academic.oup. com/poq/article/71/2/287/1928986

36 Ohlmeier C, Frick J, Prütz F, et al. [Use of routine data from statutory health insurances for federal health monitoring purposes]. Bundesgesundheitsblatt Gesundheitsforschung Gesundheitsschutz 2014:57:464-72.

37 Orme BK. Getting started with conjoint analysis: strategies for product design and pricing research. Madison, WI: Research Publishers LLC, 2010.

38 Ryan M, Kolstad JR, Rockers PC. How to conduct a discrete choice experiment for health workforce recruitment and retention in remote and rural areas: a user guide with case studies, 2012. Available: http://documents1.worldbank.org/curated/en/ 586321468156869931/pdf/NonAsciiFileName0.pdf [Accessed 15 Jun 2020].

39 Hauber AB, González JM, Groothuis-Oudshoorn CGM, et al. Statistical methods for the analysis of discrete choice experiments: a report of the ISPOR conjoint analysis good research practices Task force. Value Health 2016;19:300-15.

40 Langner I, Riedel O, Czwikla J, et al. Linkage of routine data to other data sources in Germany: a practical example illustrating challenges and solutions. Gesundheitswesen 2020;82:S117-21.

41 Jaunzeme J, Eberhard S, Geyer S. [How "representative" are $\mathrm{SHI}$ (statutory health insurance) data? Demographic and social differences and similarities between an SHI-insured population, the population of Lower Saxony, and that of the Federal Republic of Germany using the example of the AOK in Lower Saxony]. Bundesgesundheitsblatt Gesundheitsforschung Gesundheitsschutz 2013;56:447-54. 